\title{
Laboratory parameters associated with prolonged hospital length of stay in COVID-19 patients in Johannesburg, South Africa
}

\author{
J Pillai, ${ }^{1}$ MB BCh; P P K Mistry, ${ }^{1}$ MB BCh; D A le Roux, ${ }^{1}$ MB BCh; K S C Motaung, ${ }^{2}$ NDip (Biomed Tech), DTech; \\ M Mokgatle, ${ }^{3}$ BRad, PhD; P Gaylard, ${ }^{4}$ BSc, PhD; N Cengiz, ${ }^{5}$ BSc, MSc (Med); D Basu, ${ }^{6,7}$ MBBS, PhD (D) \\ ${ }^{1}$ Department of Surgery, Faculty of Health Sciences, University of the Witwatersrand, Johannesburg, South Africa \\ ${ }^{2}$ Department of Technology Transfer and Innovation, Durban University of Technology, Durban, South Africa \\ ${ }^{3}$ Department of Public Health, School of Health Care Sciences, Sefako Makgatho Health Sciences University, Pretoria, South Africa \\ ${ }^{4}$ Data Management and Statistical Analysis, Donald Gordon Medical Centre and University of the Witwatersrand, Johannesburg, South Africa \\ ${ }^{5}$ Faculty of Health Sciences, University of the Witwatersrand, Johannesburg, South Africa \\ ${ }^{6}$ Department of Public Health Medicine, Steve Biko Academic Hospital and Faculty of Health Sciences, University of Pretoria, South Africa \\ ${ }^{7}$ World Health Organization Collaborating Centre for Social Determinants of Health and Health in All Policies, Pretoria, South Africa
}

Corresponding author: D Basu (debashis.basu@up.ac.za)

Background. Coronavirus disease (COVID-19) has imposed unprecedented stressors on South Africa (SA)'s healthcare system. Superimposed on the country's quadruple burden of disease, pandemic-related care further exposes existing inequities. Some of these inequities are specific to hospital-based inpatient services, such as the geographical maldistribution of hospital beds, lack of oxygen supplies and assisted ventilation, and scarcity of trained healthcare workers. Certain high-risk groups, such as individuals with cardiometabolic comorbidity, are likely to develop severe COVID-19 disease requiring hospitalisation with potential for a prolonged length of stay (LoS). It may be helpful for health authorities to identify those at risk for prolonged $\operatorname{LoS}$ to facilitate appropriate health systems planning.

Objectives. To identify hospital admission laboratory parameters associated with a hospital stay $>14$ days in patients with COVID-19 pneumonia.

Methods. A retrospective observational study design was used. Laboratory data were obtained from an SA private laboratory for 642 inpatients with suspected or confirmed COVID-19 pneumonia, comprising 7 months of admission laboratory data from six private hospitals in Johannesburg, Gauteng Province.

Results. Of 642 hospital admissions for pneumonia, 497 were confirmed to have COVID-19 infection (reverse transcription-polymerase chain reaction test positive). In the COVID-19-positive group, hospital LoS was prolonged in $35.4 \%$ of admissions. Univariate analysis demonstrated an association with the following risk factors for prolonged LoS: older age; male sex; high serum creatinine, sodium (Na), chloride, potassium and urea levels and low estimated glomerular filtration rate; raised white blood cell count, lymphopenia, neutrophilia and an elevated neutrophil-to-lymphocyte ratio (NLR); and elevated levels of D-dimers, interleukin-6 (IL-6), and procalcitonin (PCT). The strongest univariate associations (relative risk $(\mathrm{RR}) \geq 2.0$ ) with a hospital stay $>14$ days were high Na levels, NRL $>18$, high PCT levels and IL-6 $>40 \mathrm{pg} / \mathrm{mL}$. On multivariable analysis, the following factors remained significantly associated with prolonged LoS: older age (RR 1.015 per year of age; $95 \%$ confidence interval (CI) 1.005 - 1.024); hypernatraemia (RR 1.80; 95\% CI 1.25 - 2.60); hyperkalaemia (RR 1.61; 95\% CI 1.18 - 2.20); and neutrophilia (RR 1.47; 95\% CI 1.15 - 1.88).

Conclusions. COVID-19 pandemic preparedness requires hospital-based inpatient care to be prioritised in resource-limited settings, and availability of beds and prompt admissions are essential to ensure good clinical outcomes. In this study of COVID-19 patients admitted with pneumonia, multivariable analysis showed older age, hypernatraemia, hyperkalaemia and neutrophilia to be associated with LoS $>14$ days. This may assist with healthcare systems planning.

S Afr Med J 2022;112(3):201-208. https://doi.org/10.7196/SAMJ.2022.v112i3.16024

COVID-19, caused by SARS-CoV-2, has wreaked havoc on global economies and healthcare systems around the world. The World Health Organization declared COVID-19 a pandemic in March 2020 . $^{[1]}$

Waves of new infection and new, more transmissible SARSCoV-2 variants have led to protracted lockdown regulations in many countries. ${ }^{[2-4]}$ While lockdown has been an effective method of 'flattening the curve', it is not a socially or economically sustainable solution for the long term. The ability of healthcare systems to predict and plan for surges in infection has become crucial.
The South African (SA) healthcare system is plagued by a geographical maldistribution of resources. There are 125390 general hospital beds currently available for SA's population of 60 million. ${ }^{[5]}$ Considering the country's overall high burden of disease, identification of potential risk factors or markers that predict length of hospital stay (LoS) in COVID-19 patients may be of great benefit to health authorities and may assist in triage and contingency planning.

Abnormal laboratory parameters identified at admission have been reported to predict in-hospital severity of disease and mortality. ${ }^{[6]}$ Lymphopenia, anaemia, thrombocytopenia, neutrophilia and eleva- 
ted C-reactive protein (CRP) levels have all been associated with increased severity of disease and mortality in COVID-19..$^{[7-9]}$ There is currently limited information on the value of laboratory parameters in predicting LoS in COVID-19. Reports have suggested that elevated serum aspartate aminotransferase (AST) and increased admission serum creatinine ( $\mathrm{sCr}$ ) may be associated with longer hospital stay. ${ }^{[10,11]}$

\section{Objectives}

To identify admission laboratory parameters that could potentially predict longer hospital stay in patients admitted for COVID-19 pneumonia.

\section{Methods}

\section{Study setting and design}

A retrospective, cross-sectional study design was used to conduct a secondary analysis of data from a private laboratory in Gauteng Province, SA. Data were obtained from six hospitals to which a total of 642 adults (aged $>18$ years) were admitted for the management of pneumonia during the period 8 April - 18 November 2020. All patients were admitted for suspected or confirmed COVID-19 pneumonia and all underwent reverse transcription polymerase chain reaction (RT-PCR) testing.

\section{Sample size}

Based on a $20 \%$ prevalence of risk factors, an incidence of longer LoS of $35 \%$ and the estimation of relative risk (RR) of $\geq 1.5$ with $80 \%$ power at the $5 \%$ significance level, a minimum sample size of 660 was required. ${ }^{[12]}$ The actual sample size of 642 was therefore adequate for a study of this nature.

\section{Data acquisition and variables}

We requested admission laboratory parameters of survivors only, therefore excluding patients who died during their hospital stay. As this was a secondary analysis, we did not have access to patient clinical records and we did not analyse COVID-19 symptoms, specific intensive care unit (ICU) stay or severity of illness. Laboratory staff transferred the relevant data to an Excel spreadsheet, version 2016 (Microsoft, USA), and each patient was allocated an identification number. Only de-identified data were supplied to the investigators. The various laboratory test requirements were determined by the attending clinician. We considered admission laboratory parameters to be those that were obtained during the week preceding admission, on the day of admission and 24 hours after admission.

The following variables were analysed: age, sex, LoS in days from admission to discharge, SARS-CoV-2 RT-PCR test result, serum interleukin-6 (IL-6), glycated haemoglobin (HbA1c), total serum cholesterol (TC), serum N-terminal pro b-type natriuretic peptide (NT-pro BNP), high-sensitivity cardiac troponin I, serum urea, sCr, sodium $(\mathrm{Na})$, potassium $(\mathrm{K})$, chloride $(\mathrm{Cl})$, total carbon dioxide, estimated glomerular filtration rate (eGFR), white blood cell (WBC) count, lymphocytes, neutrophils, eosinophils, platelets, neutrophilto-lymphocyte ratio (NLR), CRP, D-dimers and procalcitonin (PCT).

\section{Data analysis}

Pre-admission, admission and 24-hour post-admission laboratory data were available for some variables, and where more than one value existed, the admission values of the biomarkers were used. Laboratory data were categorised into normal, high and low according to standard reference ranges for the laboratory (Table 1). We divided IL- 6 into four categories $(<10,10-20,21-40$ and $>40$ pg/
$\mathrm{mL}$ ), as all the values were above normal. The NLR was classified into normal $(<6)$, mild $(6-<9)$, moderate $(9-18)$ and severe $(>18){ }^{[13]}$ Laboratory parameters for which $<30 \%$ of the study group were tested were excluded from analysis, i.e. HbA1c, TC, CRP and NT-pro BNP. Based on a recent review indicating that the average LoS for COVID-19 patients was 14 days, we categorised LoS as $\leq 14$ days and $>14$ days for this analysis. ${ }^{[14]}$

We compared variables in RT-PCR-positive and negative patients. The association between study variables and RT-PCR status was determined by the $\chi^{2}$ test for categorical variables (Fisher's exact test was used for $2 \times 2$ tables), and the Wilcoxon rank-sum test was used for age.

The RR of each study variable for prolonged LoS was determined, together with its $95 \%$ confidence interval (CI), using binomial regression. Categories with $n<15$ overall were not included in the analyses (no reliable inference can be made based on such small groups). For multivariable analysis, only those analytes commonly analysed together (the full blood count and urea and electrolytes groups) were considered, together with age and sex. Study variables significant at $p<0.20$ were combined into a multivariable model, after examining each pair of variables for possible confounding using the $\chi^{2}$ test (or Fisher's exact test for $2 \times 2$ tables); a value of Cramer's $\mathrm{V}$ (or the phi coefficient for Fisher's exact test) $>0.60$ was regarded as too strong an association to include both variables in a multivariable model. sCr and eGFR were strongly associated (eGFR was used), as were WBCs and neutrophils (neutrophils were used). Non-significant variables were sequentially removed from the multivariable model. These analyses were conducted for the COVID-positive group and for the whole study group. Data analysis was carried out using SAS version 9.4 for Windows (SAS Institute, USA). A 5\% significance level was used.

\section{Ethics approval}

The study was approved by the Sefako Makgatho Health Sciences University Research Ethics Committee (ref. no. SMUREC/ D/187/2020(J)).

\section{Results}

This study describes demographic and laboratory parameters of 642 patients admitted to hospital for management of pneumonia (total group). Of these, 497 patients had a positive SARS-CoV-2 RT-PCR test confirming COVID-19 infection. Comparing the total group, the RT-PCR-negative group and the RT-PCR-positive group (Table 2), $34.6 \%(n=208), 31.7 \%(n=157)$ and $35.4 \%(n=51)$, respectively, were admitted to hospital for $>14$ days; the median (interquartile range) age in the total group, the RT-PCR-negative group and the RT-PCR-positive group was $58(47-68)$ years, $59(47-71)$ years and 57 (47 - 67) years, respectively, and the relative proportion of males was $52.6 \%, 52.4 \%$ and $52.8 \%$, respectively. In terms of laboratory parameter comparison, only eosinopenia and lymphopenia were significantly prevalent in the RT-PCR-positive group compared with the RT-PCR-negative group: $62.6 \%$ v. $28.5 \%(p<0.001)$ and $46.6 \% \mathrm{v}$. $34.6 \%$ ( $p=0.0085)$, respectively. There were no significant betweengroup differences for any other parameter. A positive RT-PCR test obtained $\geq 6$ days prior to admission was associated with shorter hospital LoS for the whole group (RR 0.48 ; 95\% CI 0.25 - 0.93) (Table 2) and for those with confirmed COVID-19 infection (RR 0.31; 95\% CI 0.12 - 0.80) (Table 1).

Uni- and multivariable analysis of all admissions for pneumonia $(N=642)$ (Table 2). With univariable analysis, the following factors were significantly associated with prolonged hospital LoS: older 
Table 1. Age, sex, timing of RT-PCR test and admission laboratory parameters of the RT-PCR-positive group admitted with COVID-19 pneumonia

\begin{tabular}{|c|c|c|c|c|}
\hline Variables & $\begin{array}{l}\text { Total }(N=497), \\
n(\%)\end{array}$ & $\begin{array}{l}\leq 14 \text { days }(N=321), \\
n(\%)\end{array}$ & $\begin{array}{l}>14 \text { days }(N=176) \\
n(\%)\end{array}$ & $\begin{array}{l}\text { RR for longer LoS } \\
(95 \% \mathrm{CI})\end{array}$ \\
\hline \multicolumn{5}{|l|}{ Age (years) } \\
\hline Median (IQR) & $57(47-67)$ & $55(45-64)$ & $61(53-69)$ & $1.016(1.008-1.024)^{*}$ \\
\hline \multicolumn{5}{|l|}{ Sex } \\
\hline Female & $235(47.3)$ & $164(69.8)$ & $71(30.2)$ & 1.00 (ref.) \\
\hline Male & $262(52.7)$ & $157(59.9)$ & $105(40.1)$ & $1.33(1.04-1.69)^{\star}$ \\
\hline \multicolumn{5}{|l|}{$\begin{array}{l}\text { Days from COVID-19 test to admission } \\
(n=385)\end{array}$} \\
\hline Same day & $172(44.7)$ & $116(67.4)$ & $56(32.6)$ & 1.00 (ref.) \\
\hline 1 & $97(25.2)$ & $66(68.0)$ & $31(32.0)$ & $0.98(0.68-1.41)$ \\
\hline $2-5$ & $76(19.7)$ & $54(71.1)$ & $22(28.9)$ & $0.89(0.59-1.34)$ \\
\hline$\geq 6$ & $40(10.4)$ & $36(90.0)$ & $4(10.0)$ & $0.31(0.12-0.80)^{\star}$ \\
\hline \multicolumn{5}{|l|}{ Laboratory parameters } \\
\hline \multicolumn{5}{|l|}{$\mathrm{Na}(\mathrm{mmol} / \mathrm{L})(n=453)$} \\
\hline Low & $115(25.3)$ & $68(59.1)$ & $47(40.8)$ & $1.28(0.98-1.68)$ \\
\hline Normal (136 - 145) & $323(71.3)$ & $220(68.1)$ & $103(31.8)$ & 1.00 (ref.) \\
\hline High & $15(3.3)$ & $5(33.3)$ & $10(66.6)$ & $2.09(1.41-3.09)^{*}$ \\
\hline \multicolumn{5}{|l|}{$\mathrm{K}(\mathrm{mmol} / \mathrm{L})(n=452)$} \\
\hline Low & $38(8.4)$ & $24(63.1)$ & $14(36.8)$ & $1.11(0.72-1.73)$ \\
\hline Normal (3.5 - 5.1) & $375(82.9)$ & $251(66.9)$ & $124(33)$ & 1.00 (ref.) \\
\hline High & $39(8.6)$ & $17(43.5)$ & $22(56.4)$ & $1.71(1.25-2.33)^{*}$ \\
\hline \multicolumn{5}{|l|}{$\mathrm{Cl}(\mathrm{mmol} / \mathrm{L})(n=453)$} \\
\hline Low & $55(12.1)$ & $32(58.1)$ & $23(41.8)$ & $1.36(0.96-1.93)$ \\
\hline Normal (98 - 108) & $335(73.9)$ & $232(69.2)$ & $103(30.7)$ & 1.00 (ref.) \\
\hline High & $63(13.9)$ & $29(46)$ & $34(53.9)$ & $1.76(1.32-2.32)^{*}$ \\
\hline \multicolumn{5}{|l|}{$\mathrm{PCO}_{2}(\mathrm{mmol} / \mathrm{L})(n=452)$} \\
\hline Low & $305(67.4)$ & $191(62.6)$ & $114(37.3)$ & $1.21(0.91-1.60)$ \\
\hline Normal (22 - 28) & $142(31.4)$ & $98(69)$ & $44(30.9)$ & 1.00 (ref.) \\
\hline High & $5(1.1)$ & $3(60)$ & $2(40)$ & - \\
\hline \multicolumn{5}{|l|}{ Urea $(\mathrm{mmol} / \mathrm{L})(n=451)$} \\
\hline Low & $36(7.9)$ & $31(86.1)$ & $5(13.8)$ & $0.45(0.20-1.04)$ \\
\hline Normal $(2.9-8.2)$ & $267(59.2)$ & $185(69.2)$ & $82(30.7)$ & 1.00 (ref.) \\
\hline High & $148(32.8)$ & $75(50.6)$ & $73(49.3)$ & $1.61(1.26-2.05)^{*}$ \\
\hline \multicolumn{5}{|l|}{$\mathrm{sCr}(\mu \mathrm{mol} / \mathrm{L})(n=455)$} \\
\hline Low & $127(27.9)$ & $89(70)$ & $38(29.9)$ & $0.92(0.66-1.28)$ \\
\hline Normal (71 - 115) & $213(46.8)$ & $144(67.6)$ & $69(32.3)$ & 1.00 (ref.) \\
\hline High & $115(25.2)$ & $62(53.9)$ & $53(46)$ & $1.42(1.08-1.88)^{*}$ \\
\hline \multicolumn{5}{|l|}{$\operatorname{eGFR}(\mathrm{mL} / \mathrm{min})(n=451)$} \\
\hline Moderate to severe decrease $(<45)$ & $95(21.06)$ & $48(50.52)$ & $47(49.47)$ & $1.68(1.22-2.32)^{\star}$ \\
\hline Moderate decrease $(45-59)$ & $50(11.08)$ & $27(54)$ & $23(46)$ & $1.56(1.06-2.31)^{*}$ \\
\hline Mild decrease $(60-89)$ & $160(35.47)$ & $114(71.25)$ & $46(28.75)$ & $0.98(0.69-1.39)$ \\
\hline Normal $(\geq 90)$ & $146(32.37)$ & $103(70.54)$ & $43(29.45)$ & 1.00 (ref.) \\
\hline \multicolumn{5}{|l|}{ WBCs $\left(\times 10^{9} / \mathrm{L}\right)(n=450)$} \\
\hline Low & $48(10.66)$ & $32(66.66)$ & $16(33.33)$ & $1.10(0.71-1.69)$ \\
\hline Normal (4 - 10) & $299(66.44)$ & $208(69.56)$ & $91(30.43)$ & 1.00 (ref.) \\
\hline High & $103(22.88)$ & $52(50.48)$ & $51(49.51)$ & $1.63(1.25-2.11)^{*}$ \\
\hline \multicolumn{5}{|l|}{ Neutrophils $\left(\times 10^{9} / \mathrm{L}\right)(n=446)$} \\
\hline Low & $27(6.05)$ & $20(74.07)$ & $7(25.92)$ & $0.90(0.46-1.75)$ \\
\hline Normal $(2-7)$ & $285(63.9)$ & $203(71.22)$ & $82(28.77)$ & 1.00 (ref.) \\
\hline High & $134(30.04)$ & $67(50)$ & $67(50)$ & $1.74(1.35-2.23)^{*}$ \\
\hline \multicolumn{5}{|l|}{ Lymphocytes $\left(\times 10^{9} / \mathrm{L}\right)(n=446)$} \\
\hline Low & $199(44.61)$ & $114(57.28)$ & $85(42.71)$ & $1.49(1.16-1.93)^{\star}$ \\
\hline Normal $(1-3)$ & $245(54.93)$ & $175(71.42)$ & $70(28.57)$ & 1.00 (ref.) \\
\hline High & $2(0.44)$ & $1(50)$ & $1(50)$ & \\
\hline
\end{tabular}

Continued ... 
Table 1. (continued) Age, sex, timing of RT-PCR test and admission laboratory parameters of the RT-PCR-positive group admitted with COVID-19 pneumonia

\begin{tabular}{|c|c|c|c|c|}
\hline Variables & $\begin{array}{l}\text { Total }(N=497) \text {, } \\
n(\%)\end{array}$ & $\begin{array}{l}\leq 14 \text { days }(N=321) \\
n(\%)\end{array}$ & $\begin{array}{l}>14 \text { days }(N=176), \\
n(\%)\end{array}$ & $\begin{array}{l}\text { RR for longer LoS } \\
(95 \% \mathrm{CI})\end{array}$ \\
\hline \multicolumn{5}{|c|}{ Eosinophils $\left(\times 10^{9} / \mathrm{L}\right)(n=446)$} \\
\hline Low & $279(62.55)$ & $166(59.49)$ & $113(40.5)$ & $1.32(0.93-1.88)$ \\
\hline Normal (0.02-0.5) & $85(19.05)$ & $59(69.41)$ & $26(30.58)$ & 1.00 (ref.) \\
\hline High & $82(18.38)$ & $65(79.26)$ & $17(20.73)$ & $0.68(0.40-1.15)$ \\
\hline \multicolumn{5}{|c|}{ Platelets $\left(\times 10^{9} / \mathrm{L}\right)(n=448)$} \\
\hline Low & $66(14.73)$ & $41(62.12)$ & $25(37.87)$ & $1.10(0.79-1.55)$ \\
\hline Normal (150 - 450) & $367(81.91)$ & $241(65.66)$ & $126(34.33)$ & 1.00 (ref.) \\
\hline High & $15(3.34)$ & $8(53.33)$ & $7(46.66)$ & $1.36(0.78-2.38)$ \\
\hline \multicolumn{5}{|l|}{$\operatorname{NLR}(n=446)$} \\
\hline Normal $(<6)$ & $247(55.38)$ & $183(74.08)$ & $64(25.91)$ & 1.00 (ref.) \\
\hline Mild $(6-<9)$ & $66(14.79)$ & $40(60.6)$ & $26(39.39)$ & $1.52(1.05-2.19)^{\star}$ \\
\hline Moderate $(9-18)$ & $106(23.76)$ & $56(52.83)$ & $50(47.16)$ & $1.82(1.36-2.44)^{*}$ \\
\hline Severe $(>18)$ & $27(6.05)$ & $11(40.74)$ & $16(59.25)$ & $2.29(1.57-3.33)^{\star}$ \\
\hline \multicolumn{5}{|c|}{ D-dimers $(\mathrm{ng} / \mathrm{mL})(n=343)$} \\
\hline Normal $(\leq 0.5)$ & $72(20.99)$ & $58(80.55)$ & $14(19.44)$ & 1.00 (ref.) \\
\hline High $(>0.5)$ & $271(79)$ & $178(65.68)$ & $93(34.31)$ & $1.76(1.07-2.90)^{*}$ \\
\hline \multicolumn{5}{|l|}{ PCT $(n g / m L)(n=306)$} \\
\hline Normal (0 - 0.05) & $81(26.47)$ & $69(85.18)$ & $12(14.81)$ & 1.00 (ref.) \\
\hline High & $225(73.52)$ & $129(57.33)$ & $96(42.66)$ & $2.88(1.67-4.96)^{*}$ \\
\hline \multicolumn{5}{|c|}{ IL-6 (pg/mL) $(n=298)$ (all abnormal) } \\
\hline$<10$ & $89(29.86)$ & $77(86.51)$ & $12(13.48)$ & 1.00 (ref.) \\
\hline $10-20$ & $43(14.42)$ & $33(76.74)$ & $10(23.25)$ & $1.72(0.81-3.67)$ \\
\hline $21-40$ & $64(21.47)$ & $50(78.12)$ & $14(21.87)$ & $1.62(0.80-3.27)$ \\
\hline$>40$ & $102(34.22)$ & $60(58.82)$ & $42(41.17)$ & $3.05(1.72-5.43)^{*}$ \\
\hline \multicolumn{5}{|l|}{ HS cTnI (ng/L) $(n=225)$} \\
\hline Normal $(<34)$ & $156(72.22)$ & $148(94.87)$ & $71(45.51)$ & 1.00 (ref.) \\
\hline High $(\geq 34)$ & $60(27.77)$ & $44(73.33)$ & $35(58.33)$ & $1.25(0.88-1.79)$ \\
\hline
\end{tabular}

age, male sex, high sCr levels, high urea, high $\mathrm{K}, \mathrm{Na}$ and $\mathrm{Cl}$ levels, low eGFR $\left(<60 \mathrm{~mL} / \mathrm{min} / 1.73 \mathrm{~m}^{2}\right)$, low WBC count, lymphopenia, neutrophilia, NLR $\geq 6$, high D-dimer levels, high PCT, and IL-6 $>40 \mathrm{pg} / \mathrm{mL}$. The corresponding RRs with $95 \%$ CIs are detailed in Table 2. Multivariable analysis identified increased age (RR 1.015 per year; 95\% CI 1.008 - 1.023), male sex (RR 1.27; 95\% CI 1.01 - 1.60), hyperchloraemia (RR 1.40; 95\% CI 1.08 - 1.82) and neutrophilia (RR 1.29; 95\% CI 1.0 - 1.61) as risk factors significantly associated with prolonged hospital LoS.

Uni- and multivariable analysis for the COVID-19 group with pneumonia $(n=497)$ (Table 1). Compared with the total group, univariable analysis identified similar risk factors significantly associated with prolonged hospital LoS in the COVID-19-positive group, namely older age, male sex, high sCr levels, high urea, high $\mathrm{K}$, $\mathrm{Na}$ and $\mathrm{Cl}$ levels, low eGFR $\left(<60 \mathrm{~mL} / \mathrm{min} / 1.73 \mathrm{~m}^{2}\right)$, low WBC count, lymphopenia, neutrophilia, NLR $\geq 6$, high D-dimer levels, high PCT, and IL- $6>40 \mathrm{pg} / \mathrm{mL}$. The strongest univariate associations $(\mathrm{RR} \geq 2.0)$ with a hospital stay $>14$ days were high Na levels, NRL $>18$, high PCT levels, and IL- $6>40 \mathrm{pg} / \mathrm{mL}$. The corresponding RRs with 95\% CIs are detailed in Table 1. With multivariable analysis, older age (but not sex) (RR 1.015 per year; 95\% CI 1.005 - 1.024), hypernatraemia (RR 1.80; 95\% CI 1.25 - 2.60), hyperkalaemia (RR 1.61; 95\% CI 1.18 2.20 ) and neutrophilia (RR 1.47; 95\% CI 1.15 - 1.88) were associated with increased hospital LoS (Table 3).

\section{Discussion}

This is the first study from SA to identify factors associated with prolonged LoS in adults hospitalised for COVID-19 infection. Multivariable analysis showed that older age, high $\mathrm{Na}$, high $\mathrm{K}$ and neutrophilia were independently associated with a long hospital stay. On univariate analysis, IL- $6>40 \mathrm{pg} / \mathrm{mL}$, high PCT and high NLR correlated with an $\mathrm{RR}>2.0$. We considered all of these variables to be predictive of LoS $>14$ days.

Current reports often do not use LoS as the primary measure of outcome, and include disease severity, ICU stay or mortality as adjunctive outcomes. ${ }^{[8,15,16]}$ Alwafi et al. ${ }^{[15]}$ identified age and endstage renal failure as affecting mortality and hospital stay. A further report $^{[11]}$ indicated that abnormal liver function is common in COVID-19 and that a higher AST level is associated with a longer hospital stay. The practical implications of these findings were not clearly elucidated. ${ }^{[11]}$ A recent review indicated that LoS in patients who die of COVID-19 is shorter than in survivors. ${ }^{[14]}$ Analysing hospital stay as a primary outcome measure is therefore likely to provide salient information. In our report, we excluded patients who had died and therefore anticipated that long LoS would be likely to correlate with disease severity.

We identified that older age was associated with a hospital stay of $>14$ days. The higher prevalence of comorbid conditions in older individuals has been associated with severe COVID-19. ${ }^{[17-19]}$ 
Table 2. Age, sex, timing of RT-PCR test and admission laboratory parameters of the whole study group

\begin{tabular}{|c|c|c|c|c|}
\hline Variables & $\begin{array}{l}\text { Total }(N=642), \\
n(\%)\end{array}$ & $\begin{array}{l}\leq 14 \text { days }(N=420), \\
n(\%)\end{array}$ & $\begin{array}{l}>14 \text { days }(N=222) \\
n(\%)\end{array}$ & $\begin{array}{l}\text { RR for longer LoS } \\
(95 \% \mathrm{CI})\end{array}$ \\
\hline \multicolumn{5}{|l|}{ Age (years) } \\
\hline Median (IQR) & $58(47-68)$ & $55(45-65)$ & $63(53-71)$ & $1.016(1.009-1.023)^{*}$ \\
\hline \multicolumn{5}{|l|}{ Sex } \\
\hline Female & $304(47.35)$ & $214(70.39)$ & $90(29.6)$ & 1.00 (ref.) \\
\hline Male & $338(52.64)$ & $206(60.94)$ & $132(39.05)$ & $1.32(1.06-1.64)^{*}$ \\
\hline \multicolumn{5}{|l|}{ COVID status } \\
\hline Negative & $145(22.58)$ & $99(68.27)$ & $46(31.72)$ & 1.00 (ref.) \\
\hline Positive & $497(77.41)$ & $321(64.58)$ & $176(35.41)$ & $1.12(0.85-1.46)$ \\
\hline \multicolumn{5}{|l|}{$\begin{array}{l}\text { Days from COVID-19 test to admission } \\
(n=530)\end{array}$} \\
\hline Same day & $260(49.05)$ & $181(69.61)$ & $79(30.38)$ & 1.00 (ref.) \\
\hline 1 & $129(24.33)$ & $86(66.66)$ & $43(33.33)$ & $1.097(0.808-1.489)$ \\
\hline $2-5$ & $86(16.22)$ & $57(66.27)$ & $29(33.72)$ & $1.109(0.783-1.572)$ \\
\hline$\geq 6$ & $55(10.37)$ & $47(85.45)$ & $8(14.54)$ & $0.48(0.25-0.93)^{\star}$ \\
\hline \multicolumn{5}{|l|}{ Laboratory parameters } \\
\hline \multicolumn{5}{|l|}{$\mathrm{Na}(\mathrm{mmol} / \mathrm{L})(n=585)$} \\
\hline Low & $156(26.66)$ & $93(59.61)$ & $63(40.38)$ & $1.29(1.02-1.64)^{*}$ \\
\hline Normal (136 - 145) & $413(70.59)$ & $284(68.76)$ & $129(31.23)$ & 1.00 (ref.) \\
\hline High & $16(2.73)$ & $6(37.5)$ & $10(62.5)$ & $2.00(1.33-3.00)^{\star}$ \\
\hline \multicolumn{5}{|l|}{$\mathrm{K}(\mathrm{mmol} / \mathrm{L})(n=584)$} \\
\hline Low & $50(8.56)$ & $32(64)$ & $18(36)$ & $1.11(0.75-1.65)$ \\
\hline Normal (3.5 - 5.1) & $482(82.53)$ & $326(67.63)$ & $156(32.36)$ & 1.00 (ref.) \\
\hline High & $52(8.9)$ & $24(46.15)$ & $28(53.84)$ & $1.66(1.25-2.21)^{*}$ \\
\hline \multicolumn{5}{|l|}{$\mathrm{Cl}(\mathrm{mmol} / \mathrm{L})(n=585)$} \\
\hline Low & $73(12.47)$ & $42(57.53)$ & $31(42.46)$ & $1.39(1.03-1.88)^{*}$ \\
\hline Normal (98 - 108) & $429(73.33)$ & $298(69.46)$ & $131(30.53)$ & 1.00 (ref.) \\
\hline High & $83(14.18)$ & $43(51.8)$ & $40(48.19)$ & $1.58(1.21-2.06)^{\star}$ \\
\hline \multicolumn{5}{|l|}{$\mathrm{PCO}_{2}(\mathrm{mmol} / \mathrm{L})(n=584)$} \\
\hline Low & $400(68.49)$ & $254(63.5)$ & $146(36.5)$ & $1.20(0.93-1.56)$ \\
\hline Normal (22 - 28) & $178(30.47)$ & $124(69.66)$ & $54(30.33)$ & 1.00 (ref.) \\
\hline High & $6(1.02)$ & $4(66.66)$ & $2(33.33)$ & - \\
\hline \multicolumn{5}{|l|}{ Urea $(\mathrm{mmol} / \mathrm{L})(n=583)$} \\
\hline Low & $47(8.06)$ & $39(82.97)$ & $8(17.02)$ & $0.57(0.30-1.09)$ \\
\hline Normal $(2.9-8.2)$ & $344(59)$ & $241(70.05)$ & $103(29.94)$ & 1.00 (ref.) \\
\hline High & $192(32.93)$ & $101(52.6)$ & $91(47.39)$ & $1.58(1.27-1.97)^{\star}$ \\
\hline \multicolumn{5}{|l|}{$\mathrm{sCr}(\mu \mathrm{mol} / \mathrm{L})(n=587)$} \\
\hline Low & $173(29.47)$ & $126(72.83)$ & $47(27.16)$ & $0.83(0.62-1.13)$ \\
\hline Normal (71 - 115) & $261(44.46)$ & $176(67.43)$ & $85(32.56)$ & 1.00 (ref.) \\
\hline High & $153(26.06)$ & $83(54.24)$ & $70(45.75)$ & $1.40(1.10-1.80)^{*}$ \\
\hline \multicolumn{5}{|l|}{$\operatorname{eGFR}(\mathrm{mL} / \mathrm{min})(n=580)$} \\
\hline Moderate to severe decrease $(<45)$ & $127(21.89)$ & $67(52.75)$ & $60(47.24)$ & $1.77(1.31-2.39)^{\star}$ \\
\hline Moderate decrease (45 - 59) & $64(11.03)$ & $31(48.43)$ & $33(51.56)$ & $1.93(1.38-2.70)^{\star}$ \\
\hline Mild decrease $(60$ - 89) & $202(34.82)$ & $145(71.78)$ & $57(28.21)$ & $1.06(0.76-1.46)$ \\
\hline Normal $(\geq 90)$ & $187(32.24)$ & $137(73.26)$ & $50(26.73)$ & 1.00 (ref.) \\
\hline \multicolumn{5}{|l|}{ WBCs $\left(\times 10^{9} / \mathrm{L}\right)(n=583)$} \\
\hline Low & $56(9.6)$ & $38(67.85)$ & $18(32.14)$ & $1.04(0.69-1.57)$ \\
\hline Normal $(4-10)$ & $392(67.23)$ & $271(69.13)$ & $121(30.86)$ & 1.00 (ref.) \\
\hline High & $135(23.15)$ & $75(55.55)$ & $60(44.44)$ & $1.44(1.13-1.83)^{*}$ \\
\hline \multicolumn{5}{|l|}{ Neutrophils $\left(\times 10^{9} / \mathrm{L}\right)(n=576)$} \\
\hline Low & $30(5.2)$ & $22(73.33)$ & $8(26.66)$ & $0.91(0.49-1.68)$ \\
\hline Normal (2 - 7) & $371(64.4)$ & $262(70.61)$ & $109(29.38)$ & 1.00 (ref.) \\
\hline High & $175(30.38)$ & $96(54.85)$ & $79(45.14)$ & $1.54(1.22-1.93)^{\star}$ \\
\hline
\end{tabular}

Continued ... 
Table 2. (continued) Age, sex, timing of RT-PCR test and admission laboratory parameters of the whole study group

\begin{tabular}{|c|c|c|c|c|}
\hline Variables & $\begin{array}{l}\text { Total }(N=642), \\
n(\%)\end{array}$ & $\begin{array}{l}\leq 14 \text { days }(N=420), \\
n(\%)\end{array}$ & $\begin{array}{l}>14 \text { days }(N=222), \\
n(\%)\end{array}$ & $\begin{array}{l}\text { RR for longer LoS } \\
(95 \% \mathrm{CI})\end{array}$ \\
\hline \multicolumn{5}{|c|}{ Lymphocytes $\left(\times 10^{9} / \mathrm{L}\right)(n=576)$} \\
\hline Low & $244(42.36)$ & $142(58.19)$ & $102(41.8)$ & $1.47(1.17-1.84)^{*}$ \\
\hline Normal $(1-3)$ & $326(56.59)$ & $233(71.47)$ & $93(28.52)$ & 1.00 (ref.) \\
\hline High & $6(1.04)$ & $5(83.33)$ & $1(16.66)$ & \\
\hline \multicolumn{5}{|c|}{ Eosinophils $\left(\times 10^{9} / \mathrm{L}\right)(n=576)$} \\
\hline Low & $316(54.86)$ & $193(61.07)$ & $123(38.92)$ & $1.21(0.90-1.63)$ \\
\hline Normal $(0.02-0.5)$ & $115(19.96)$ & $78(67.82)$ & $37(32.17)$ & 1.00 (ref.) \\
\hline High & $145(25.17)$ & $109(75.17)$ & $36(24.82)$ & $0.77(0.52-1.14)$ \\
\hline \multicolumn{5}{|c|}{ Platelets $\left(\times 10^{9} / \mathrm{L}\right)(n=580)$} \\
\hline Low & $76(13.1)$ & $45(59.21)$ & $31(40.78)$ & $1.23(0.91-1.65)$ \\
\hline Normal $(150$ - 450) & $484(83.44)$ & $323(66.73)$ & $161(33.26)$ & 1.00 (ref.) \\
\hline High & $20(3.44)$ & $13(65)$ & $7(35)$ & $1.05(0.57-1.94)$ \\
\hline \multicolumn{5}{|l|}{$\operatorname{NLR}(n=576)$} \\
\hline Normal $(<6)$ & $324(56.25)$ & $238(73.45)$ & $86(26.54)$ & 1.00 (ref.) \\
\hline Mild $(6-<9)$ & $79(13.71)$ & $50(63.29)$ & $29(36.7)$ & $1.38(0.98-1.95)$ \\
\hline Moderate $(9-18)$ & $136(23.61)$ & $76(55.88)$ & $60(44.11)$ & $1.66(1.28-2.16)^{*}$ \\
\hline Severe $(>18)$ & $37(6.42)$ & $16(43.24)$ & $21(56.75)$ & $2.14(1.53-2.99)^{*}$ \\
\hline \multicolumn{5}{|c|}{ D-dimers $(\mathrm{ng} / \mathrm{mL})(n=427)$} \\
\hline Normal $(\leq 0.5)$ & $94(22.01)$ & $76(80.85)$ & $18(19.14)$ & 1.00 (ref.) \\
\hline High $(>0.5)$ & $333(77.98)$ & $227(68.16)$ & $106(31.83)$ & $1.66(1.07-2.59)$ \\
\hline \multicolumn{5}{|l|}{ PCT $(\mathrm{ng} / \mathrm{mL})(n=395)$} \\
\hline Normal $(0-0.05)$ & $108(27.34)$ & $93(86.11)$ & $15(13.88)$ & 1.00 (ref.) \\
\hline High & $287(72.65)$ & $168(58.53)$ & $119(41.46)$ & $2.99(1.83-4.87)^{\star}$ \\
\hline \multicolumn{5}{|c|}{ IL-6 $(\mathrm{pg} / \mathrm{mL})(n=383)$ (all abnormal) } \\
\hline$<10$ & $125(32.63)$ & $111(88.8)$ & $14(11.2)$ & 1.00 (ref.) \\
\hline $10-20$ & $56(14.62)$ & $45(80.35)$ & $11(19.64)$ & $1.75(0.85-3.62)$ \\
\hline $21-40$ & $74(19.32)$ & $59(79.72)$ & $15(20.27)$ & $1.81(0.93-3.53)$ \\
\hline$>40$ & $128(33.42)$ & $78(60.93)$ & $50(39.06)$ & $3.487(2.03-5.98)^{\star}$ \\
\hline \multicolumn{5}{|l|}{ HS cTnI (ng/L) $(n=298)$} \\
\hline Normal $(<34)$ & $219(73.48)$ & $148(67.57)$ & $71(32.42)$ & 1.00 (ref.) \\
\hline High $(\geq 34)$ & $79(26.51)$ & $44(55.69)$ & $35(44.3)$ & $1.37(1.00-1.87)$ \\
\hline
\end{tabular}

RT-PCR = reverse transcription polymerase chain reaction; $\mathrm{RR}=$ relative risk; $\mathrm{LoS}=$ length of stay; $\mathrm{CI}=$ confidence interval; $\mathrm{IQR}=$ interquartile range; $\mathrm{Na}=$ sodium; $\mathrm{K}=$ potassium; $\mathrm{Cl}=$ chloride; $\mathrm{PCO}_{2}=$ partial pressure of carbon dioxide; $\mathrm{sCr}=$ serum creatinine; eGFR = estimated glomerular filtration rate; $\mathrm{WBCs}=$ white blood cells; $\mathrm{NLR}=$ neutrophil-to-lymphocyte ratio;

PCT = procalcitonin; IL- $6=$ interleukin- $6 ; \mathrm{HS}$ cTnI $=$ high-sensitivity cardiac troponin I.

Table 3. Multivariable parameters in the RT-PCR-positive group $(N=432)$

\begin{tabular}{lll}
\hline Variable & Category & RR (95\% CI for RR) \\
\hline Age & n/a & $1.015 /$ year $(1.005-1.024)$ \\
$\mathrm{Na}(\mathrm{mmol} / \mathrm{L})$ & High $>145$ & $1.80(1.25-2.60)$ \\
$\mathrm{K}(\mathrm{mmol} / \mathrm{L})$ & High $>5.1$ & $1.61(1.18-2.20)$ \\
Neutrophils $\left(\times 10^{9} / \mathrm{L}\right)$ & High $>7$ & $1.47(1.15-1.88)$ \\
& &
\end{tabular}

However, exaggerated immune responses, increased intravascular coagulation, decreased physiological capacity, decreased functional reserve and declining immunity are all associated with increasing age and severe COVID-19. ${ }^{[17,18]}$

We found that an NLR of $>18$ was associated with a hospital stay of $>14$ days. The NLR has been used as a marker of inflammation and may be predictive of clinical outcomes in systemic illnesses such as cardiovascular disease, cancer and pancreatitis. ${ }^{[13,16,20,21]}$ A systematic review of the NLR in COVID-19 $9^{[22]}$ and an article by Cavalcante-Silva et al. ${ }^{[23]}$ confirmed its predictive value in identifying severe cases early on. Similarly, neutrophils have been associated with severe COVID19 and poor outcomes. ${ }^{[24]}$ Studies indicate that neutrophil activation and degranulation are processes that occur in severe acute respiratory syndrome and may enhance antiviral defences. ${ }^{[23,24]}$

In the present study, high PCT was associated with a hospital stay of $>14$ days. PCT is a propeptide of calcitonin, which is usually produced by the thyroid tissue and is not associated with hormonal activity. ${ }^{[25,26]}$ During bacterial infection, PCT may be produced by extra-thyroid tissue and acts as a mediator of inflammation. ${ }^{[25,26]}$ It has been shown that in severe COVID-19, PCT may be markedly 
elevated. ${ }^{[25,26]}$ It has also been shown that PCT may remain within the normal range in non-complicated SARS-CoV-2 infection, so it is generally accepted that a substantial increase in PCT is associated with bacterial co-infection and portends a more complex clinical outcome. ${ }^{[25,26]}$ In the present study, $61.6 \%$ of the COVID-19-positive patients had an admission PCT test. Of these, $73.5 \%$ were high (Table 1). Routine admission PCT testing may not only assist in predicting outcomes, but also guide the early investigation and treatment of bacterial infections. This requires further investigation.

One of the most commonly measured cytokines in symptomatic COVID-19 is IL-6. ${ }^{[27-29]}$ We found that IL- $6>40 \mathrm{pg} / \mathrm{mL}$ was associated with a hospital stay $>14$ days. In addition to the prognostic value of IL-6, an elevated level of $>10 \mathrm{pg} / \mathrm{mL}$ has been associated with the cytokine storm. ${ }^{[27-29]}$ The airway epithelium is one of the first lines of defence in SARS-CoV-2 infection. A complex mechanism results in the release of cytokines (e.g. IL-6) by activated alveolar macrophages, thereby triggering an inflammatory response. ${ }^{[27-29]}$ Phagocytic disruption is thought to be associated with the cytokine storm. ${ }^{[27-29]}$ In the present study, $60 \%$ of patients who were COVID19-positive had an admission IL-6 test. Of these, 34\% fell into the $>40 \mathrm{pg} / \mathrm{mL}$ range. The value of routine admission IL- 6 testing and its predictive value in various COVID-19 outcomes requires evaluation in a larger study.

We found that high $\mathrm{Na}$ levels were associated with a hospital stay $>14$ days. Dysnatraemia in COVID-19 hospitalised patients has generally been associated with poorer outcomes. ${ }^{[30-42]}$ Both hypernatraemia and hyponatraemia, at admission, have been associated with increased mortality, ICU admission, longer hospitalisation and mechanical ventilation. ${ }^{[22,37,38]}$ Specifically, hyponatraemia was found to be relatively common in hospitalised COVID-19 patients and to be associated with severe illness and increased in-hospital mortality. ${ }^{[3,41,42]}$ On the contrary, admission hypernatraemia or the development of hypernatraemia during hospitalisation have been associated with increased mortality and death. ${ }^{[30,35,36,39,40]}$ Similarly, we found a correlation between hypernatraemia and a long hospital stay. It has been reported that SARS-CoV-2 binds to the angiotensin-converting enzyme 2 receptor in kidney tubules. ${ }^{[30,31]}$ The resulting high levels of angiotensin II may facilitate $\mathrm{Na}$ reabsorption, leading to hypernatraemia. ${ }^{[0,31]}$ In the context of admission hypernatraemia in COVID-19, it is currently uncertain what the role is of dehydration, advanced age and diarrhoea. This requires further investigation. However, low admission $\mathrm{Na}$ levels have also been reported in severe COVID19. ${ }^{[32,33]}$ Hypotheses implicate the syndrome of inappropriate antidiuretic hormone secretion, intestinal injury and acute kidney injury. ${ }^{[32,33]}$ Larger studies are required to analyse the role and predictive value of dysnatraemia in subsets of COVID-19 patients. Efforts to identify and correct dysnatraemia and other electrolyte imbalances as early as possible are prudent. These may potentially improve outcomes and also require further investigation.

Similar to dysnatraemia, high and low K levels have been associated with COVID-19. ${ }^{[43-45]}$ We identified high $\mathrm{K}$ to be associated with a hospital stay $>14$ days. In an editorial, Chan et al. ${ }^{[44]}$ reported the relatively common occurrence of hyperkalaemia in hospitalised COVID-19 patients and those with severe disease.

The prolonged SARS-CoV-2 pandemic has resulted in the need for healthcare workers to predict bed capacity and to accommodate regular patients. Using laboratory parameters to predict LoS seems intuitive and requires further validation in the context of hospital protocols.

\section{Study limitations}

This study was conducted in the private healthcare sector and in a relatively small number of hospitals in Gauteng, which limits generalisability of the results. In addition, these data pertain to admissions for pneumonia, and in the absence of additional clinical data further inferences regarding the patients' profile and severity of illness are limited. We were only able to analyse laboratory parameters that were supplied by the laboratory, and there was inevitable heterogeneity in the number of tests per patient. The diverse prevalence of laboratory tests for similar patient populations is usual in clinical situations and is based on physician preference. Nonetheless, it could have been a source of reporting bias, and other parameters may be predictive of LoS. A large study analysing these clinical symptoms, COVID-19 severity and ICU stay, together with biomarkers, would be helpful.

\section{Conclusions}

COVID-19 and the development of new strains of SARS-CoV-2 have disrupted healthcare systems around the world. Availability of beds and prompt admission, particularly during peaks of infection, are essential to ensure good clinical outcomes. In this study we identified older age, high $\mathrm{Na}$, high $\mathrm{K}$ and high neutrophils (multivariable analysis) and NLR $>18$, high PCT and IL- $6>40 \mathrm{pg} / \mathrm{mL}$ (RR $>2.0$ univariate analysis) to be predictive of a hospital stay $>14$ days. This information may help health service providers to triage and better manage bed occupancy. Our findings that NLR, Na, K and neutrophils were predictive of LoS are relevant in the context of early bed allocation and resource planning. These parameters are almost routinely requested upon hospital admission, and are easily available and relatively inexpensive.

\section{Declaration. None.}

Acknowledgements. The authors express their appreciation to Jan Vermaak and his staff for supplying the laboratory data. They are also grateful to the Wits Donald Gordon Medical Centre Research Office for logistical support and for funding the statistical analysis.

Author contributions. Conception/design of the work: JP, DB. Data collection/verification: JP, PPKM, DAlR, KSCM, NC, MM. Data analysis and interpretation: PG, JP. Drafting the article: JP, NC. Critical revision of the article: JP, PG. Final approval of the version to be published: JP, DB.

Funding. None.

Conflicts of interest. None.

1. World Health Organization. WHO coronavirus (COVID-19) dashboard. 2021. https://covid19.who. int/table (accessed 4 June 2021)

2. Xuefei R. Pandemic and lockdown: A territorial approach to COVID-19 in China, Italy and the United States. Euras Geog Econ 2020;61(4-5):423-434. https://doi.org/10.1080/15387216.2020.1762103

Haider N, Osman AY, Gadzekpo A, et al. Lockdown measures in response to COVID-19 in Haider N, Osman AY, Gadzekpo A, et al. Lockdown measures in response to COVID-19 in
nine sub-Saharan African countries. BMJ Glob Health 2020;5:e003319. https://doi.org/10.1136/ nine sub-Saharan

4. Karim SSA, de Oliveira T. New SARS-CoV-2 variants - clinical, public health, and vaccine implications. N Engl J Med 2021;384(19):1866-1868. https://doi.org/10.1056/NEJMc2100362

5. Dell AJ, Kahn D. Geographical maldistribution of surgical resources in South Africa: A review of the number of hospitals, hospital beds and surgical beds. S Afr Med J 2017;107(12):1099-1105. https://doi. org/10.7196/SAMJ.2017.v107i12.12539

6. Wolff D, Nee S, Hickey N, Marschollek M. Risk factors for COVID-19 severity and fatality: A structure literature review. Infection 2020;49(1):15-28. https://doi.org/10.1007/s15010-020-01509-1

Bhargava A, Fukushima E, Levine M, et al. Predictors for severe COVID-19 infection. Clin Infect Dis 2020;71(8):1962-1968. https://doi.org/10.1093/cid/ciaa674

8. Zhang L, Yan X, Fan Q, et al. D-dimer levels on admission to predict in-hospital mortality in patient with Covid-19. J Thromb Haemost 2020;18(6):1324-1329. https://doi.org/10.1111/jth.14859

9. Malik $\mathrm{P}$ Patel U Mehta D, et al. Biomers and outcomes of COVID-19 hopitalisation Malik P, Patel U, Mehta D, et al. Biomarkers and outcomes of COVID-19 hospitalisation Systematic review and

10. Guo A, Lu J, Tan H, et al. Risk factors on admission associated with hospital length of stay in patient with COVID-19: A retrospective cohort study. Sci Rep 2021;11:7310. https://doi.org/10.1038/s41598021-86853-4 
11. Gu X, Li X, An X, et al. Elevated serum aspartate aminotransferase level identifies patients with coronavirus disease 2019 and predicts the length of hospital stay. J Clin Lab Anal 2020;34(7):e23391 coronavirus disease 2019 and predct
https://doi.org/10.1002/jcla.23391

12. Woodward M. Epidemiology: Study Design and Data Analysis. 3rd ed. London: Chapman \& Hall/ CRC, 2013.

13. Xiaojie L, Hui L, Zhongcheng G, Chenggang W, Yaqi N. The predictive value of interleukin- 6 and neutrophil-lymphocyte ratio in patients with severe and extremely severe oral and maxillofacial space infections. Biomed Res Int 2021;2021:2615059. https://doi.org/10.1155/2021/2615059

14. Rees EM, Nightingale ES, Jafari Y, et al. COVID-19 length of hospital stay: A systematic review and data synthesis. BMC Med 2020;18:270. https://doi.org/10.1186/s12916-020-01726-3

15. Alwafi H, Naser AY, Qanash S, et al. Predictors of length of hospital stay, mortality, and outcomes among hospitalised COVID-19 patients in Saudi Arabia: A cross-sectional study. J Multidiscip Healthc 2021;15(14):839-852. https://doi.org/10.2147/JMDH.S304788

16. Li X, Liu C, Mao Z, et al. Predictive values of neutrophil-to-lymphocyte ratio on disease severity and mortality in COVID-19 patients: A systematic review and meta-analysis. Crit Care 2020;24(647):1-10. https://doi.org/10.1186/s13054-020-03374-8

17. Ahrenfeldt LJ, Otavova M, Christensen K, Lindahl-Jacobsen R. Sex and age differences in COVID-19

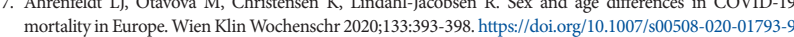
18. Davies NG, Klepac P, Liu Y, et al. Age-dependent effects in the transmission and control of COVID-19 epidemics. Nature Med 2020;26:1205-1211. https://doi.org/10.1038/s41591-020-0962-9

19. Worldometer. Age, sex, existing conditions of COVID-19 cases and deaths. 13 May 2020. https://www. worldometers.info/coronavirus/coronavirus-age-sex-demographics/ (accessed 7 June 2021).

20. Kim S, Eliot M, Koestler DC, Wu WC, Kelsey KT. Association of neutrophil-to-lymphocyte ratio with mortality and cardiovascular disease in the Jackson heart study and modification by the Duffy antigen variant. JAMA Cardiol 2018;3(6):455-462. https://doi.org/10.1001/jamacardio.2018.1042

21. Ponti G, Maccaferri M, Ruini C, Tomasi A, Ozben T. Biomarkers associated with COVID-19 disease progression. Crit Rev Clin Lab Sci 2020;57(6):389-399. https://doi.org/10.1080/10408363.2020.1770685

22. Templeton AJ, McNamara MG, Šeruga B, et al. Prognostic role of neutrophil-to-lymphocyte ratio in solid tumors: A systematic review and meta-analysis. J Nat Cancer Inst 2014;106(6):dju124. https:// solid tumors: A systematic
doi.org/10.1093/inci/dju124

23. Cavalcante-Silva L, Carvalho D, Lima ÉA, et al. Neutrophils and COVID-19: The road so far. Int Immunopharmacol 2021;90:107233. https://doi.org/10.1016/.intimp.2020.107233

24. Dennison D, Al Khabori M, Al Mamari S, et al. Circulating activated neutrophils in COVID-19: An independent predictor for mechanical ventilation and death. Int J Infect Dis 2021;106(1):155-159. https://doi.org/10.1016/j.ijid.2021.03.066

25. Schuetz P. The role of procalcitonin for risk assessment and treatment of COVID-19 patients. HealthManagement 2020;20(5):380-382

26. Vijayan AL, Vanimaya SR, Saikant R, et al. Procalcitonin: A promising diagnostic marker for sepsis and antibiotic therapy J Intensive Care 2017:5(51):1-7. https.//doi.org/10.1186/40560-017-0246-8

27. Copaescu A, Smibert O, Gibson A, Phillips EJ Trubiano IA. The role of IL-6 and other mediators in the cytokine storm associated with SARS-CoV-2 infection. J Allergy Clin Immunol 2020;146(3):518-534. https://doi.org/10.1016/.jaci.2020.07.001

28. Gubernatorova EO, Gorshkova EA, Polinova AI, Drutskaya MS. IL-6: Relevance for immunopathology 8. Gubernatorova EO, Gorshkova EA, Polinova AI, Drutskaya MS. IL-6: Relevance for immunopathology
of SARS-CoV-2. Cytokine Growth Factor Rev 2020;53(1):13-24. https://doi.org/10.1016/j. of SARS-CoV-2.
cytogfr.2020.05.009

29. Coomes EA, Haghbayan H. Interleukin-6 in Covid-19: A systematic review and meta-analysis. Rev Med Virol 2020;30(6):1-9. https://doi.org/10.1002/rmv.2141
30. Sjöström A, Rysz S, Sjöström H, et al. Hypernatremia is common in patients with severe COVID-19 and indicates a poor prognosis. Res Square 2020;1-17. https://doi.org/10.21203/rs.3.rs-81965/v1

31. Zimmer MA, Zink AK, Weißer CW, et al. Hypernatremia - a manifestation of COVID-19: A case series. A\&A Pract 2020;14(9):e01295. https://doi.org/10.1213/XAA.0000000000001295

32. Ruiz-Sánchez JG, Núñez-Gil II, Cuesta M, et al. Prognostic impact of hyponatremia and hypernatremia in COVID-19 pneumonia: A HOPE-COVID-19 (Health Outcome Predictive Evaluation for COVID-19) registry analysis. Front Endocrinol 2020;11:599255. https://doi.org/10.3389/ fendo.2020.59925

33. Gheorghe G, Ilie M, Bungau S, Stoian AMP, Bacalbasa N, Diaconu CC. Is there a relationship between COVID-19 and hyponatremia? Medicina (Kaunas) 2021;57(1):55. https://doi.org/10.3390/ medicina 57010055

34. Hu W, Lv X, Li C, et al. Disorders of sodium balance and its clinical implications in COVID-19 patients: A multicenter retrospective study. Intern Emerg Med 2021;16(4):853-862. https://doi. org/10.1007/s11739-020-02515-9

35. Voets PJ, Frölke SC, Vogtländer NP, Kaasjager KA. COVID-19 and dysnatremia: A comparison between COVID-19 and non-COVID-19 respiratory illness. SAGE Open Med 2021;9:20503121211027778. https://doi.org/10.1177/20503121211027778

36. Hirsch JS, Uppal NN, Sharma P, et al. Prevalence and outcomes of hyponatremia and hypernatremia in patients hospitalized with COVID-19. Nephrol Dial Transplant 2021;36(6):1135-1138. https://doi. in patients hospitalized with
org/10.1093/ndt/gfab067

37. Atila C, Sailer CO, Bassetti S, et al. Prevalence and outcome of dysnatremia in patients with COVID-19 compared to controls. Eur J Endocrinol 2021;184(3):409-418. https://doi.org/10.1530/EJE-20-1374

38. Pourfridoni M, Abbasnia SM, Shafaei F, Razaviyan J, Heidari-Soureshjani R. Fluid and electrolyte disturbances in COVID-19 and their complications. Biomed Res Int 2021;2021:6667047. https://doi. $\operatorname{org} / 10.1155 / 2021 / 6667047$

39. Tzoulis P, Waung JA, Bagkeris E, et al. Dysnatremia is a predictor for morbidity and mortality in hospitalized patients with COVID-19. J Clin Endocrinol Metab 2021;106(6):1637-1648. https://doi. org/10.1210/clinem/dgab107

40. Martino M, Falcioni P, Giancola G, et al. Sodium alterations impair the prognosis of hospitalized patients with COVID-19 pneumonia. Endocr Connect 2021:10(10):1344-1351. https://doi. org/10.1530/EC-21-0411

41. Berni A, Malandrino D, Corona G, et al. Serum sodium alterations in SARS CoV-2 (COVID-19) infection: Impact on patient outcome. Eur J Endocrinol 2021;185(1):137-144. https://doi.org/10.1530/ EJE-20-1447

42. Saad G, Abdelkrim AB, El Abed YH, et al. Disorders of sodium balance in COVID-19 patients: Two Tunisian patients report. Pan Afr Med J 2021:39:199. https://doi.org/10.11604/pamj.2021.39.199.27626

43. Socha V. Rates of hyperkalemia in patients with AKI and COVID-19. Nephrology Times, 16 December 2020. https://www.docwirenews.com/nephtimes/nephtimes-diseases-and-conditions/hyperkalemia/ rates-of-hyperkalemia-in-patients-with-aki-and-covid-19/ (accessed 7 June 2021).

44. Chan L, Hindi J, Nadkarni GN. COVID-19: The kidneys tell a tale. Am J Kidney Dis 2021;77(2):175177. https://doi.org/10.1053/j.ajkd.2020.11.001

45. Gaetano A, Annachiara F, Francesco F, et al. Hypokalemia in patients with COVID-19. Clin Exp Nephrol 2021;25(4):401-409. https://doi.org/10.1007/s10157-020-01996-4

Accepted 29 November 2021. 